\title{
Hemangioma cavernoso gigante de seno maxilar. Reporte de un caso y revisión de la literatura
}

\section{Giant cavernous hemangioma of the maxillary sinus. Case report and literature review}

Alfredo Naser $\mathrm{G}^{1}$, Arturo Samith $\mathrm{M}^{1}$, Carlos Ríos $\mathrm{D}^{2}$.

\begin{abstract}
RESUMEN
Los hemangiomas son neoplasias vasculares benignas que se presentan de preferencia en la región cervicofacial, siendo raros en las fosas nasales y excepcionales en los senos paranasales, con pocos casos reportados. Se presenta un paciente de sexo masculino y 33 años de edad, que consultó por obstrucción nasal, aumento de volumen maxilar y exoftalmo izquierdo de 2 años de evolución. La tomografía computada con contraste mostraba una masa hipodensa con realce anular del contraste en maxilar izquierdo, con extensión a la cavidad nasal, con destrucción del piso de la órbita y la pared anterior del seno maxilar. Se tomó biopsia que se informó como hemangioma cavernoso, por lo que fue manejado con embolización y escleroterapia arterial supraselectiva previa a la resección. Se realizó una maxilectomía subtotal por abordaje de Weber-Ferguson, sin recidiva al seguimiento a 15 meses. Además se comparan los hallazgos y el manejo de nuestro paciente con revisiones extranjeras.
\end{abstract}

Palabras clave: Hemangioma cavernoso, tumor vascular, tumor de seno maxilar.

\begin{abstract}
Hemangiomas are benign vascular neoplasms that occur preferentially in the head and neck, being uncommon in the nasal cavities and exceptionally rare in the paranasal sinuses, with very few cases reported. We present the case of a 33 year old male, that consulted for nasal obstruction, maxillary enlargement and left exophthalmos of 2 years of evolution. Contrast enhanced computed tomography showed a hypodense mass with a ring enhanced lesion in the left maxilla, extending to the nasal cavity, with orbital floor and anterior wall of the maxillary sinus destruction. A biopsy sample was informed as cavernous hemangioma. Accordingly, it was treated by supraselective arterial embolization and sclerotherapy previous to surgical resection. A subtotal maxillectomy was performed following the Weber-Ferguson approach, with no recidives after a 15 month follow-up. In addition, we compare our findings and patient management with those reviewed in the literature.
\end{abstract}

Key words: Cavernous hemangioma, vascular tumor, maxillary sinus tumor.

Médico Otorrinolaringólogo, Servicio de Otorrinolaringología, Hospital Clínico de la Universidad de Chile.

2 Médico Cirujano, Universidad de Chile. 


\section{INTRODUCCIÓN}

Los hemangiomas son neoplasias vasculares benignas, se presentan con más frecuencia en la infancia y pueden presentarse en cualquier parte del cuerpo. En 1982, Mulliken y Glowacki propusieron una clasificación de lesiones vasculares, distinguiendo tumores vasculares de malformaciones vasculares basados en la apariencia clínica, características histopatológicas y comportamiento biológico. Los hemangiomas fueron identificados como tumores vasculares que experimentan una fase de crecimiento activo caracterizado por proliferación endotelial e hipercelularidad seguido por una fase de involución gradual que ocurre tras varios años. En contraste, las malformaciones vasculares fueron descritas como congénitas derivadas de la vasculatura de los capilares, venas, linfáticos, arterias o una combinación de éstos ${ }^{1}$. En 1996 esta clasificación fue modificada en el consenso de la sociedad internacional para el estudio de anomalías vasculares; expandiendo la categoría de tumores vasculares para incluir a los hemangioendoteliomas kaposiformes, angiomas en penacho, granulomas piógenos y hemangiopericitomas; que fueron clasificados como hemangiomas, pero demostraron tener distintas características clínicas e histopatológicas: siendo actualmente este sistema la clasificación estándar usada en el campo de anomalías vasculares². Además, Batsakis los clasificó histológicamente de acuerdo al tipo de vasculatura involucrada en la lesión; dependiendo del tamaño de los vasos que predominan en el tumor y los describió como: 1) capilares, que son frecuentemente vistos en la niñez y tienden a involucionar espontáneamente; 2) cavernosos, son frecuentes en adultos y no desarrollan involución espontánea; y 3) mixtos ${ }^{3}$. A la fecha, la patogénesis del hemangioma es poco conocida.

La región cervicofacial es un sitio común para la formación de hemangiomas en adultos siendo los labios, mejillas, frente y cuello los lugares más frecuentemente afectados ${ }^{4}$; cuando se presentan en la cavidad nasal, $85 \%$ es en el tabique nasal anterior y $15 \%$ en la pared lateral ${ }^{5}$; el tipo histológico predominante es capilar ${ }^{6,7}$. Son excepcionales en senos paranasales con pocos casos reportados a nivel mundial siendo la mayoría de tipo caverno- $\mathrm{so}^{8-12}$. Es por ello que presentamos este caso clínico, donde analizamos su manejo y realizamos una revisión de la literatura.

\section{CASO CLÍNICO}

Paciente de sexo masculino de 33 años de edad, sin antecedentes de importancia, que consulta por cuadro de 2 años de evolución, caracterizado por obstrucción nasal persistente, bilateral de predominio izquierdo, e infecciones sinusales recurrentes que cedían sólo parcialmente a tratamientos antibióticos adecuados. Al momento de consultar también presentaba algia facial izquierda, epifora, proptosis ipsilateral con movilidad ocular conservada, y aumento de volumen malar izquierdo de 2 meses de evolución. En la rinoscopía anterior de fosa nasal izquierda se observó desplazamiento hacia medial de la pared del seno maxilar con mucosa conservada. Se solicitó tomografía computada (TC) con contraste de cavidades perinasales, donde se visualizó lesión voluminosa que parecía originarse en la pared posterior del seno maxilar, con densidad de partes blandas (Figura 1), con realce anular del medio de contraste (Figuras 2a y 2b), y que protruye hacia la cavidad nasal, con desviación de la línea media y destrucción ósea (Figura 3).

Con la clínica y los hallazgos radiológicos descritos, se plantea el diagnóstico de tumor de

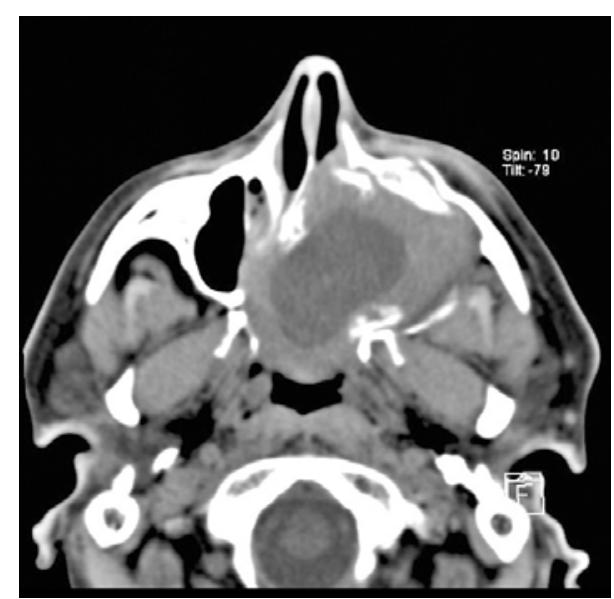

Figura 1. Tomografía computada con lesión expansiva del maxilar izquierdo. 


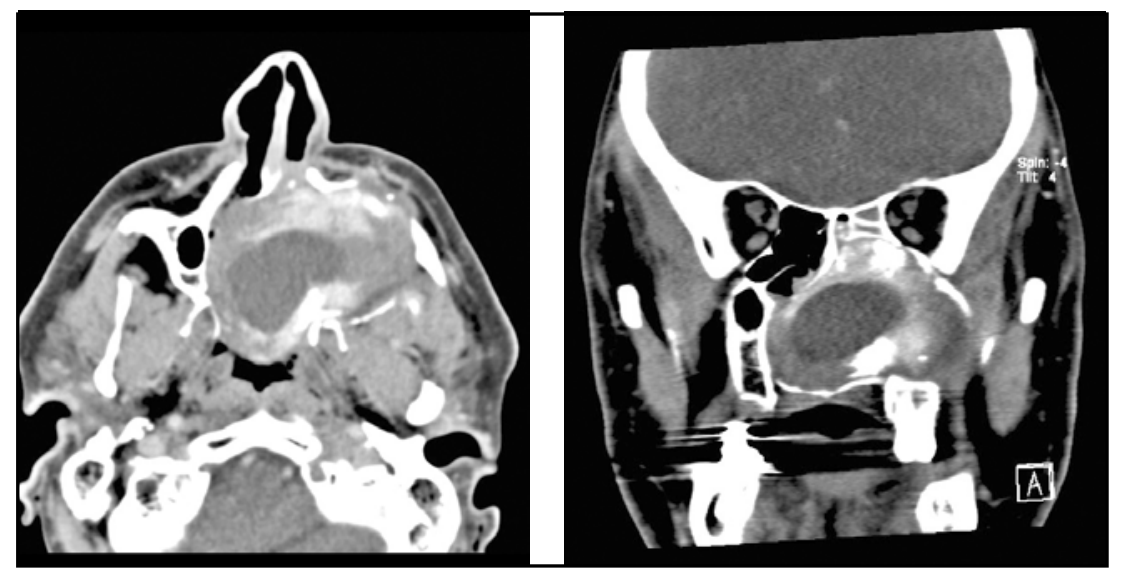

Figura 2a. TC (corte axial) con realce anular al medio de contraste, que delimita cavidad hipodensa central. $2 \mathrm{~b}$. TC corte coronal con realce del medio de contraste.

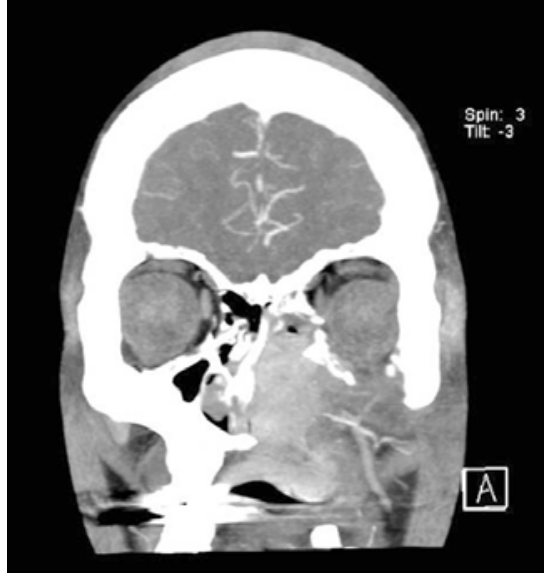

Figura 3. TC corte coronal evidencia destrucción ósea de piso de órbita y pared anterior maxilar.

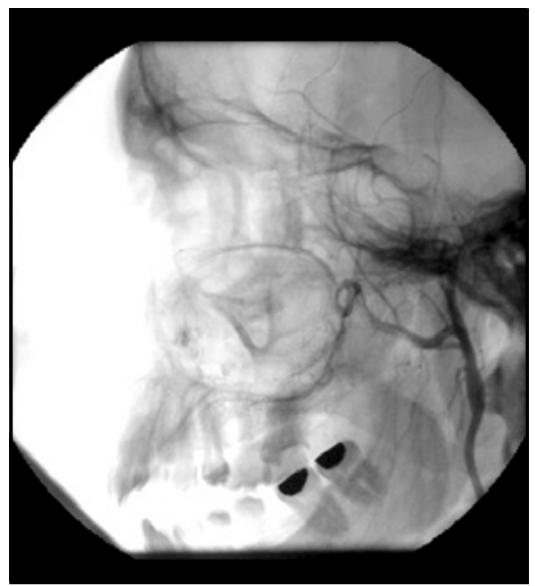

Figura 4. Angiografía: tumor vascularizado dependiente de arteria maxilar izquierda. seno maxilar, planteando inicialmente, que pudiese tratarse de un piomucocele, por lo que se planificó biopsia y posible drenaje en pabellón bajo anestesia general. Se intentó realizar el procedimiento por vía endoscópica produciéndose un sangrado profuso, que requirió complementar la técnica endoscópica con un abordaje vía Caldwell Luc para tomar una muestra de biopsia representativa y optimizar el control del sangrado, que cedió con un taponamiento posterior ipsilateral. En el posoperatorio evolucionó con anemia severa que se corrigió con transfusiones, sin presentar sangrado posterior. La biopsia se informó como hemangioma cavernoso. Once días después de la biopsia se realizó angiografía por vía femoral que mostró tumor maxilar izquierdo moderadamente vascularizado con aferentes de la arteria maxilar izquierda (Figura 4) que se embolizó en forma superselectiva. Tres días después de la embolización se intentó retirar el taponamiento posterior en pabellón, pero nuevamente se produce una hemorragia importante que requiere la reinstalación del taponamiento, se decide esclerosar la lesión de manera percutánea bajo visión angiográfica.

A los 5 días de realizada la escleroterapia se realizó la maxilectomía total por rinotomía lateral, sin incidentes. Una semana después se colocó prótesis obturadora sin dificultades. Se le dio el alta 15 días posmaxilectomía; la biopsia definitiva corroboró el diagnóstico de hemangioma cavernoso. Se le realizó TC poscirugía que evidenció tejido 
fibrogranuloso remanente, sin residuos de tumor (Figura 5). Al examen físico se evidenciaba una leve depresión infraorbitaria izquierda con movimientos oculares normales; a los 6 meses de la cirugía se realizó la reconstrucción del piso de la órbita con prótesis de titanio, con lo que mejoró su apariencia estética (Figura 6). Los controles hasta los 15 meses después de la cirugía no evidencian recidiva tumoral.

\section{DISCUSIÓN}

Los hemangiomas son lesiones endoteliales benignas que usualmente se ven en piel, mucosas y sistema esquelético; siendo la variante capilar mucho más frecuente que la cavernosa en localización nasosinusal5; así Fu y Perzin al estudiar 256 tumores no epiteliales, de cavidad nasal, senos paranasales y nasofaringe, encontraron 85 de estirpe vascular, entre ellos 30 hemangiomas capilares localizados en la parte anterior del tabique nasal, en menor número en cornetes y vestíbulo, encontrando sólo 2 casos a nivel maxilar ${ }^{14}$. También, Sciarreta y cols, dentro de su revisión de tumores benignos nasosinusales ${ }^{15}$ describen 13 casos de tumores vasculares, ninguno de ellos en seno maxilar ${ }^{16}$.

La mayoría de los hemangiomas tienen su origen primario en la mucosa que cubre al seno o la cavidad nasal, que es el sitio donde probablemente se inició en nuestro paciente. Los hemangiomas nasales son de tamaño limitado y pueden ser fácilmente detectados, mientras que los de cavidades perinasales, por su localización, son habitualmente diagnosticados cuando ya han formado una gran masa; por lo que los síntomas más frecuentemente reportados son epistaxis recurrente, obstrucción nasal, edema, dolor de mejilla y protrusión de globo ocular $^{17,18}$ (por destrucción ósea local); síntomas menos frecuentes como hemoptisis también han sido reportados ${ }^{19}$. Llama la atención que nuestro paciente no presentó epistaxis de manera espontánea, signo casi invariablemente presente en los casos reportados.

Dentro del diagnóstico diferencial de tumores en seno maxilar deben incluirse los papilomas invertidos, neuromas, osteomas, displasia fibrosa, angiofibroma juvenil, mucocele y tumores malignos de cualquier estirpe ${ }^{20,21}$; y dentro de los tumo-

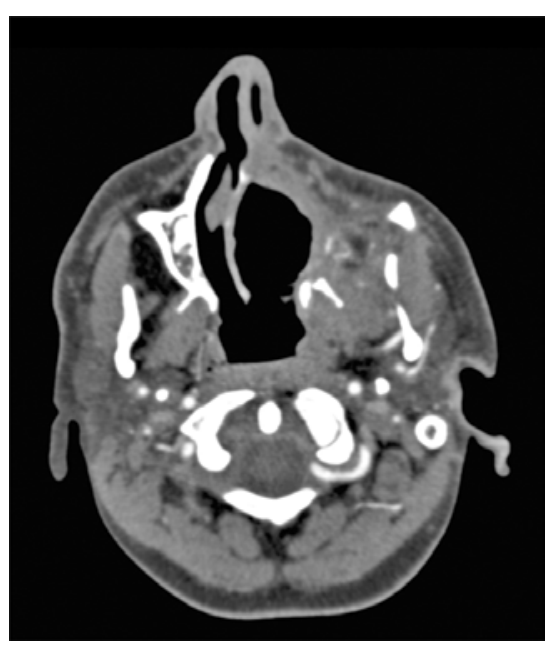

Figura 5. TC de control a los 8 meses poscirugía.

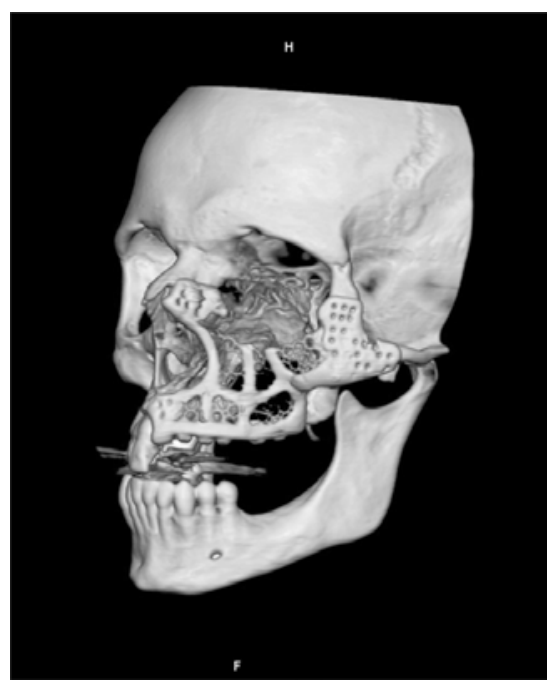

Figura 6. Reconstrucción tridimensional de TC posoperatoria con prótesis de titanio.

res vasculares, los hemangioendoteliomas (cuyo sitio de presentación es el seno maxilar en el 50\% de casos reportados ${ }^{22}$ ), los hemangiomas óseos ${ }^{23}$ y quistes óseos aneurismáticos ${ }^{24}$, deberían también estar presentes.

La TC y la resonancia magnética (RM) son usadas para delinear de mejor manera las estructuras vitales y la extensión tumoral al tejido blando, aunque su rol preoperatorio puede ser limitado $0^{17,25}$; la TC usualmente revela un aumento de la vascularización del tumor con el contraste; en 
algunos reportes se han descrito signos de erosión y destrucción ósea causada por este tipo de tumores, causando en algunos casos un diagnóstico erróneo de malignidad ${ }^{12,21,26,27}$; por su parte la RM demuestra una señal hiperintensa en T2. Ninguno de estos hallazgos es patognomónico; aunque algunos casos sugieren que la presencia de nódulos calcificados (flebolitos) dentro de la lesión podría considerarse como un signo muy sugerente de hemangioma ${ }^{28}$; sin embargo en la actualidad se prefiere a la angio-TC con reconstrucción tridimensional (3D), puesto que evidencia con más detalle las lesiones vasculares de cabeza y cuello, mostrando casi perfectamente la anatomía de este tipo de lesiones complejas ${ }^{29}$.

En cuanto al manejo, la mayoría de los reportes sugieren que la embolización de la arteria principal del tumor vascular debería ser realizado antes de la resección quirúrgica; siendo sus ventajas la disminución del tamaño tumoral y del riesgo de hemorragia durante el procedimiento quirúrgico ${ }^{11,18,19}$. En el tratamiento de la sinusitis crónica y de tumores benignos de cavidades perinasales, la cirugía endoscópica funcional ha reemplazado en la actualidad al abordaje abierto por su probada seguridad y efectividad; existiendo casos de hemangiomas maxilares de tamaño mediano resecados por vía endoscópica sin dificultades, con la ventaja de preservación de mucosa sana y estructuras óseas $18,19,25,27$. Esto no fue posible en nuestro paciente por el tamaño de la masa y la destrucción ósea ya existente; por lo que las ventajas de la vía endoscópica ya no fueron factibles y la vía de abordaje seleccionada fue la de Weber-Ferguson con resección total de la lesión sin presentar recidiva a 15 meses de seguimiento.

Los hemangiomas deberían ser considerados en el diagnóstico diferencial de tumores de seno maxilar. Ninguno de los signos, síntomas o resultados imagenológicos son patognomónicos de esta patología; aunque la presencia de flebolitos podría orientarnos en el diagnóstico de este tipo de lesiones. Una valoración angiográfica con embolización previa a la resección es lo más indicado; siendo el abordaje quirúrgico abierto 0 endoscópico individualizado, en función de la accesibilidad, extensión de la lesión, edad del paciente y consideraciones estéticas.

\section{BIBLIOGRAFÍA}

1. Mulliken JB, Glowack J. Hemangiomas and vascular malformations in infants and children: a classification based on endothelial characteristics. Plast Reconstr Surg 1982; 69: 412-22.

2. Adams D, Lucky A. Cervicofacial vascular anomalies. Hemangiomas and other benign vascular tumors. Semin Pediatr Surg 2006; 15(2): 124-32.

3. BatSAKIS J. Tumors of the Head and Neck: Clinical and Pathological Considerations. Baltimore: Editorial Williams and Wilkins; 1979: 291-312.

4. KAng G, Song C. Forty one cervicofacial vascular anomalies and their surgical treatmentRetrospection and Review. Ann Acad Med Singapore 2008; 37(3): 165-79.

5. Caylakli F, Can A, Hürcan C, Bal N, Kizllkilic 0, KIROgLU F. Cavernous hemangioma of the middle turbinate: A case report. ENT 2008; 87(1): 391-3.

6. Puxeddu R, Berlucchi M, Ledda GP, Parodo G, Farina D, Nicolal P. Lobular capillary hemangioma of the nasal cavity: A retrospective study on 40 patients. Am J Rhinol 2006; 20: 480-4.

7. Iwata N, Hattori $K$, Nakagawa $T$, Tsujimura $T$. Hemangioma of the nasal cavity: a clinicopathologic study. Auris Nasus Larynx 2002; 29: 335-9.

8. Sheppard L, Mickelson S. Hemangiomas of the nasal septum and paranasal sinuses. Henry Ford Hosp Med J 1990; 38(1): 25-7.

9. Lahoz M, Galvé A, Royo J, Valero J, Cámara F. Hemangioma cavernoso del seno maxilar. Acta Otorrinolaringol Esp 1990; 41(3): 197-9.

10. Amor J, Juiz P, Zubiarreta A. Hemangioma cavernoso del seno maxilar. Acta Otorrinolaring Esp 1998; 49(2): 165-7.

11. Raboso E, Rosell A, Plaza G, Martinez-Vidal A. Haemangioma of the maxillary sinus. $J$ Laryngol Otol 1997; 111: 638-40.

12. Engels $T$, Schörner W, Felix $R$, Witt H, Jahnke V. Cavernous hemangioma of the maxillary sinus. HNO 1990; 38(9): 342-4.

13. Dufour H, Fesselet J, Métellus P, FigarellaBranger D, Grisoli F. Cavernous hemangioma of 
the sphenoid sinus: Case report and review of the literature. Surg Neurol 2001; 55: 169-73.

14. Fu Y, Perzin K. Non epithelial tumors of the nasal cavity, paranasal sinuses and nasopharynx: a clinicopathologic study. Cancer 1974; 33: 1275-88.

15. Sciarretta V, Pasquini E, Frank G, Modugno G, Cantaroni C, Mazzatenta D, Farneti $G$. Endoscopic treatment of benign tumors of the nose and paranasal sinuses: A report of 33 cases. Am J Rhinol 2006; 20(1): 64-71.

16. Sciarretta V, Pasquini E, Farneti G, Frank $G$, Mazzatenta D, Calbucci F. Endoscopic sinus surgery for the treatment of vascular tumors. Am J Rhinol 2006; 20: 426-31.

17. Walch C, Anderhuber W, LuXenberger W, Humer-Fuchs $U$. Cavernous haemangioma in the infraorbital nerve canal: An unusual expansion en the maxillary sinus. J Laryngol Otol 1998; 112: 872-4.

18. Kanazana $T$, Inou $R$, Ohta $Y$, Watanabe $Y$, Iino $Y$. Maxillary haemangioma succesfully resected by endoscopic approach. J Laryngol Otol 2008; 1-3.

19. Jammal H, Barakat F, Hadi U. Maxillary sinus cavernous hemangioma: A rare entity. Acta Otolaryngol 2004; 124: 331-3.

20. Eviatar E, Vaiman M, Shlamkovitch N, Segal S, Kessler A, Katzenell U. Removal of Sinonasal Tumors by the Endonasal Endoscopic Approach. IMAJ 2004; 6: 346-9.

21. Pasquini E, Sciarretta V, Frank G, Cantaroni C, Modugno G, et al. Endoscopic treatment of benign tumors of the nose and paranasal sinuses. Otolaryngol Head Neck Surg 2004; 131: 180-6.
22. Semino L, Pagella F, Delú G, Todeschini A, Luinetti 0 , Zappol $F$ et aL. Endoscopic treatment of ethmoidal hemangioendothelioma: case report and review of the literature. Am J Otolaryngol 2006; 27(2): 287-90.

23. Goyal $N$, Jones $M$, Sandison A, Clarke P. Maxillary haemangioma. J Laryngol Otol 2006; 120: e14.

24. Guzmán P, Baeza A, Araya J, Roa J, Brevis L, TORRES P. Quiste óseo aneurismático del maxilar superior. Reporte de un caso. Rev Méd Chile 2005; 133: 1355-60.

25. Morales M, Sanjurjo J, Madrigal J. Hemangioma cavernoso del seno maxilar: reporte de un caso y revisión de la literatura. An Orl Mex 2005; 50(2): 26-9.

26. KIm HJ, KIm JH, KIm JH, Hwang EG. Bone erosion caused by sinonasal hemangioma: $\mathrm{CT}$ findings in two patients. Am J Neuroradiol 1995; 16(5): 1176-8.

27. Mussak E, Lin J, Prasad M. Hemangioma cavernous of maxillary sinus with bone erosion. ENT 2007; 86(9): 565-6.

28. Altug H, BüyüKsoy V, ОKcu K, Dogan N. Hemangiomas of the head and neck with phleboliths: Clinical features, diagnostic imaging and treatment of 3 cases. Oral Surg Oral Med Oral Pathol Oral Radiol Endod 2007; 103: e60-e64.

29. Perkins J, Sidhu M, Manning S, Ghioni V, Sze R. Three-dimensional CT angiography imaging of vascular tumors of the head and neck. Int J Pediatr Otolaryngol 2005; 69(3): 319-25.

Dirección: Dr. Alfredo Naser González

Santos Dumont 999. Recoleta. Santiago. Chile

E mail: aanaser@gmail.com 